\title{
Novel Green Synthesis and Characterization of Nanopolymer Porous Gold Oxide Nanoparticles
}

\author{
Manal A Awad ${ }^{1 \star}$, Khalid MO Ortashi ${ }^{2}$, Awatif A Hendi ${ }^{3 \star}$, Nada E Eisa ${ }^{4}$ and \\ Fatimah Al-Abbas ${ }^{5}$ \\ ${ }^{1}$ King Abdullah Institute for Nanotechnology, ${ }^{2}$ Department of Chemical Engineering, ${ }^{3}$ Physics Department, King Saud \\ University, Riyadh, ${ }^{4}$ Department of Physics, University of Dammam, Dammam, ${ }^{5}$ Department of Chemistry, College of Science, \\ King Saud University, Riyadh, Saudi Arabia
}

*For correspondence: Email: ahindi@ksu.edu.sa, a.manalawad@gmail.com

Received: 13 March 2015

Revised accepted: 30 August 2015

\begin{abstract}
Purpose: To develop a novel approach to green synthesis of nano-polymer porous gold oxide nanoparticles, and examine the effects of the temperatures on their surface.

Methods: Green synthesis of nano-polymer porous gold oxide nanoparticles (GONPs) using cetyle trimethylammonium bromide (CTAB) surfactant with a mixture of Olea europaea fruit and Acacia Nilotica extracts, was performed using sol-gel method. The nanoporous particles were characterized by UV (ultraviolet (UV) visible spectroscopy and dynamic light scattering (DLS) while a zetasizer was applied to determine their average particle size. Their surface morphology and shape were assessed by transmission electron microscopy (TEM) and scanning election microscopy (SEM) while surface area was measured using nitrogen gas adsorption method.

Results: TEM and SEM images showed a smooth, cylindrical or spherical, and cluster shapes, and porous surface morphology. Increase in calcination temperature resulted in increase in surface area and pore volume of nanoparticles. This feature yielded GONPs that were unique with a high surface area of $146.706 \mathrm{~m}^{2} / \mathrm{g}$.

Conclusion: The approach used in this study constitutes a new and rapid green synthesis of porous nanoparticles of gold oxide under simple conditions. Furthermore, increase in GONPs surface area is enhanced by increase in calcination temperature.
\end{abstract}

Keywords: Gold oxide, Nanoporous, Green synthesis, Olea europaea, Acacia Nilotica, Surface area, Nanopolymer, Surface morphology

Tropical Journal of Pharmaceutical Research is indexed by Science Citation Index (SciSearch), Scopus, International Pharmaceutical Abstract, Chemical Abstracts, Embase, Index Copernicus, EBSCO, African Index Medicus, JournalSeek, Journal Citation Reports/Science Edition, Directory of Open Access Journals (DOAJ), African Journal Online, Bioline International, Open-J-Gate and Pharmacy Abstracts

\section{INTRODUCTION}

In recent years, nanomaterials have been a core focus of nanoscience and nanotechnology: an ever-growing multidisciplinary field of study attracting tremendous interest, investment and effort in research and development around the world. Nanoporous materials as a subset of nanostructured materials, possess unique surface, structural, and bulk properties that underline their important uses in various fields such as ion exchange, separation, catalysis, sensor, biological molecular isolation and purifications. The presence of pores (holes) in a material has useful properties that do not exist in bulk material. Many inorganic nanoporous materials are made of oxides. They have: a high surface to volume ratio, a high surface area and large porosity, and a very ordered uniform pore 
structure. They are often non-toxic, inert, and chemically and are thermally stable [1].

Gold is an inert metal with high chemical stability due to its high surface-to-volume ratio. This enhances physical and chemical characteristics not possible with the bulk material and thus attracts distinct attention from the scientific and industrial communities. It has been used for catalytic oxidation of $\mathrm{CO}[2,3]$ as well as production of: electrodes of fuel cells [4], chemical sensors and biosensors $[5,6]$ and as a low-temperature heat exchanger [7]. Controllable pore morphology of np-Au provides a highly adaptable system for the fundamental study of wide range of mechanical and surface properties [8] in addition to a number of studies on various aspects of $\mathrm{np}-\mathrm{Au}$, such as its catalytic and optical applications [9], There are still many underexplored features of this material, including surface functionalization via thiol-conjugate chemistry and biological applications [10].

There are several methods for preparing nanoporous materials, such as electrochemical erosion [11] electrochemical oxidation/chemical reduction processes [12], chemical dealloying of alloys $[5,7,13,14]$, electrochemical alloying/ dealloying $[4,15,16]$ and (the complicated) template synthesis [17-19].

The aim of this study is to present a novel green synthesis of gold oxide nanoporous particles (GONPs) using surfactant CTAB with mix of Olea europaea fruit and Acacia nilotica extracts and few drops of sodium chloride under high temperature; and study their characterization. The preparation of GONPs by this approach has advantages in physical and chemical approaches since it produces a new type of high functional GONPs which is eco-friendly, economic, clean, nontoxic, simple to apply highly stable. To the best of our knowledge, this is the first study that discusses preparation of gold oxide nanoporous using this method.

\section{EXPERIMENTAL}

The approach entailed the production of green nano-polymer porous gold oxide nanoparticles (GONPs) as follows: GONPs were synthesized by adding $7 \mathrm{ml}$ of aqueous solution of $(5: 5) \mathrm{mix}$ of Olea europaea fruit and Acacia nilotica extracts to the aqueous solution of: $0.25 \mathrm{~mole} / \mathrm{L}$ colloidal chloroauric acid (HAuCl4), 0.05 mole/L cetyle trimethylammonium bromide (CTAB) under high temperature: $350{ }^{\circ} \mathrm{C}$ for $10 \mathrm{~min}$, and finally adding 3 drops of sodium chloride. The mixture was then dried in air to get GONPs. The products were calcined for $4 \mathrm{~h}$ at $400,500,600,700{ }^{\circ} \mathrm{C}$. Gold oxide nanoporous structure was observed by TEM. SEM and the surface area were measured by BET method.

\section{Characterization of nanopolvmer porous gold oxide}

Green synthesis of nanopolymer porous gold oxide nanoparticles was characterized using:

A UV visible spectroscopy analysis of porous GONPs was recorded as a function of time at room temperature using Perkin Elmer UV- visible spectrometer Lambda 25 (PerkinElmer. United Kingdom). The electrical conductivity and the average size of synthesized GONPs were analyzed using dynamic light scattering (DLS) Zetasizer. Nano series. HT Laser, ZEN3600 from Molvem Instrument, UK. The samples, in dried state, were characterized using elemental analysis on a single particle which confirms the elemental gold oxide using Oxford Instrument.

Incax-act energy dispersive spectrometer (EDS) analysis equipped with scanning electron microscopy (SEM, Jeol- Fesem) was used to characterize the shape and morphology of the biogenic, synthesized GONPs. Transmission electron microscopy (TEM) JEM-1011, (JEOL. Japan) was used to characterize the size, shape and morphology of the resulting biosynthesized GONPs. This was prepared by drop of GONPs solution on carbon coated copper grid with a dry film. TEM measurement was performed at an accelerated voltage of $100 \mathrm{KY}$. Specific surface area, pore volume and average pore diameter were measured with TriStar II 3020 VI.03, (Micromeritics, USA). Specific surface area of the samples was measured by adsorption of nitrogen gas at $150^{\circ} \mathrm{C}$ applying Brunauer Emmett Teller (BET) model. Prior to adsorption, the samples were automatically degassed. Pore size distributions were derived from desorption isotherms at $\mathrm{P} / \mathrm{Po}$ of 0.3 using Barrett JoynerHalenda (BJH) method.

\section{RESULTS}

In liquid state, the sample had absorbance peak (SPR) in the visible range at $577.06 \mathrm{~nm}$; the sharp peak indicates that the particles are monodispersed (Figure 1).

The particles size of the nanoparticles green synthesized was variable and had an average value of $36 \mathrm{~nm}$. Zeta potential shows the electrical properties of nano-polymer porous gold oxide, which has electrical conductivity of 6 
$\mathrm{mS} / \mathrm{cm}$. In our method the zeta potential was 26 $\mathrm{mV}$ with positively charged nanoparticles.

Transmission electron microscopy (TEM) shows that the nano- polymer porous gold oxide green synthesized has cylindrical, rod and cluster shapes as shown in below (Figure 2).

SEM was used to characterize the surface morphology of nano-polymer porous gold oxide. Several bright cylindrical, rod-like, spherical, and cluster-shaped particles were found (Figure 3 ).
Table 1 shows the elements (\%) in the green nanoparticle suspension.

Table 1: Elemental composition of the GONPs

\begin{tabular}{lcc}
\hline Element & Weight (\%) & Atomic (\%) \\
\hline $\mathrm{O}, \mathrm{K}$ & 17.90 & 69.50 \\
$\mathrm{Zn}, \mathrm{K}$ & 4.99 & 4,94 \\
$\mathrm{Au}, \mathrm{M}$ & 77.82 & 25.56 \\
Total & $\mathbf{1 0 0 . 7 1}$ & $\mathbf{1 0 0}$ \\
\hline
\end{tabular}

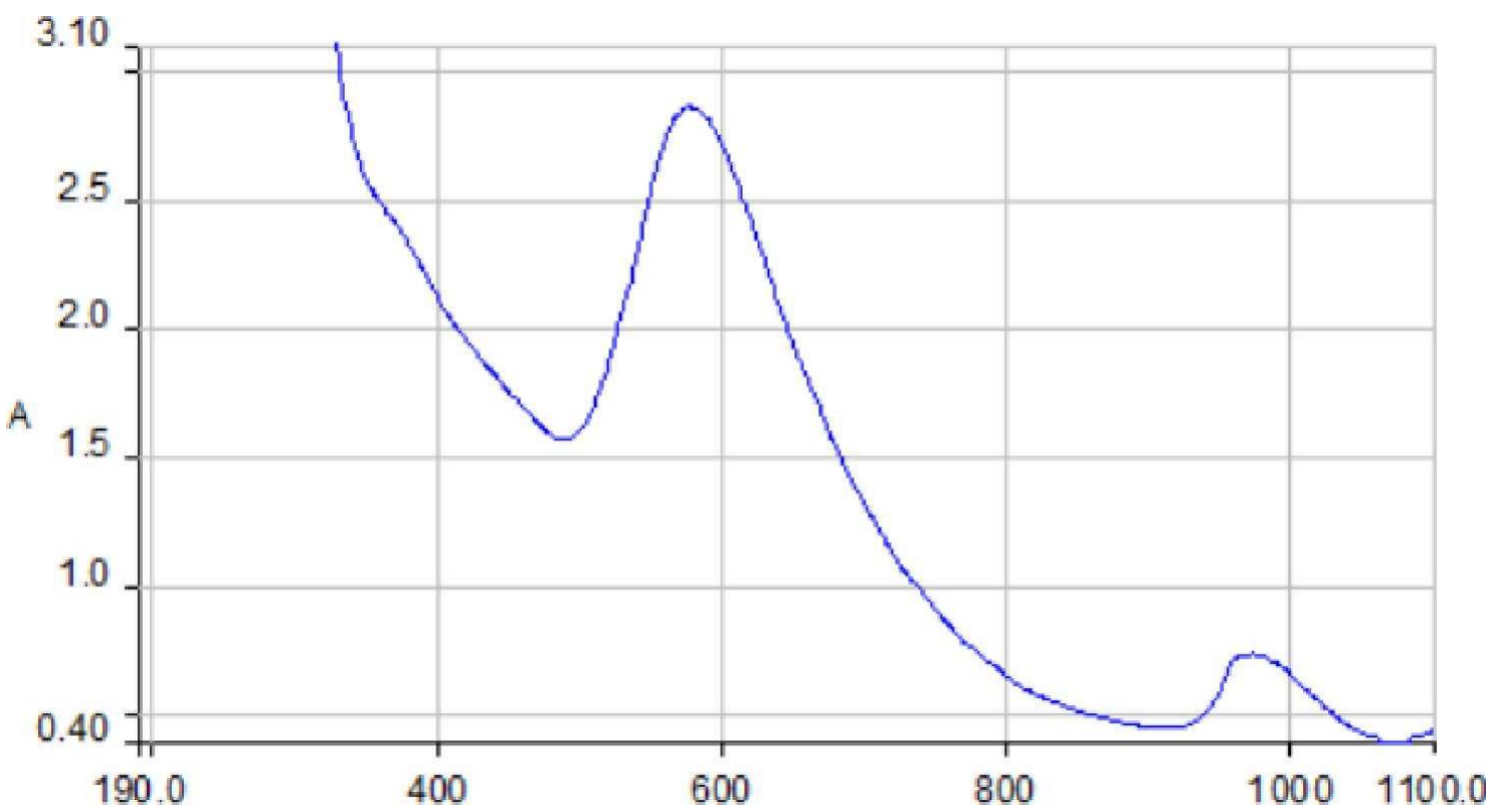

Figure 1: UV-Vis absorption spectrum of the GONPs

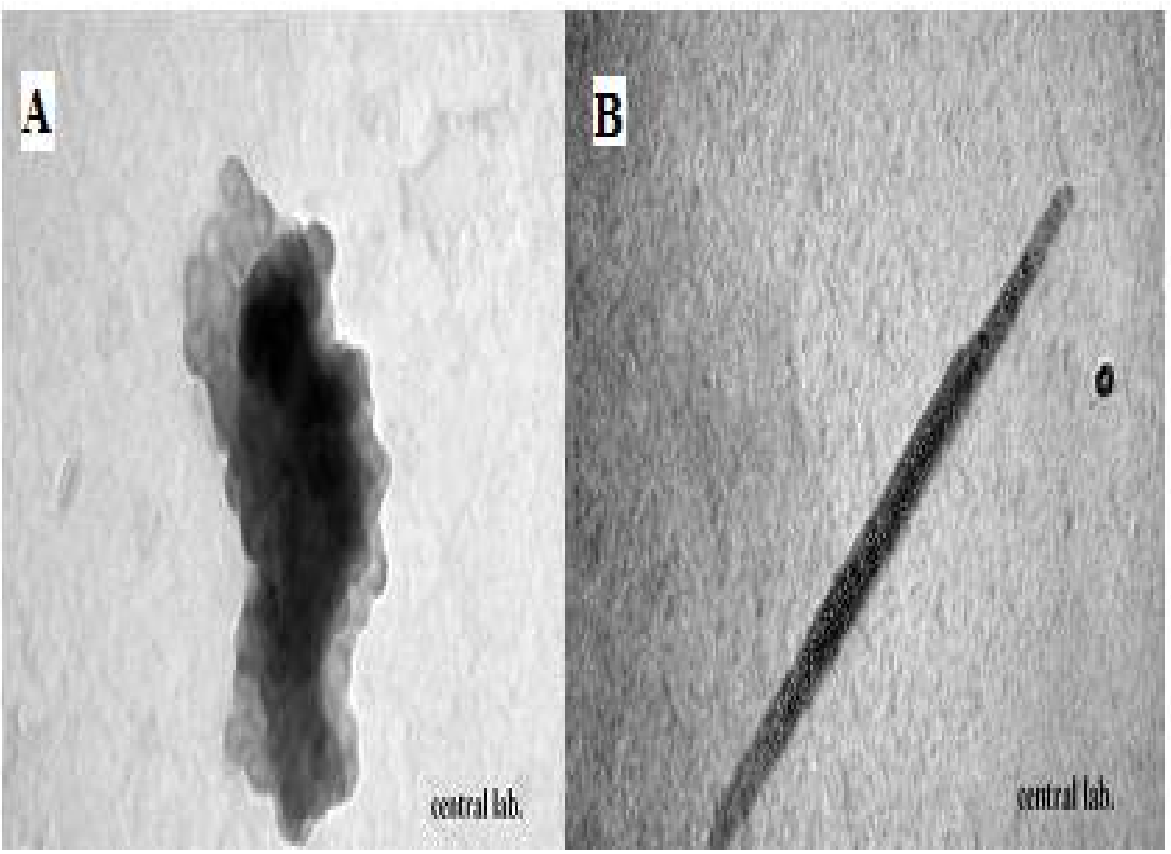

Figure 2: TEM images of GONPs (A) $100 \mathrm{~nm}$; (B) $200 \mathrm{~nm}$ 


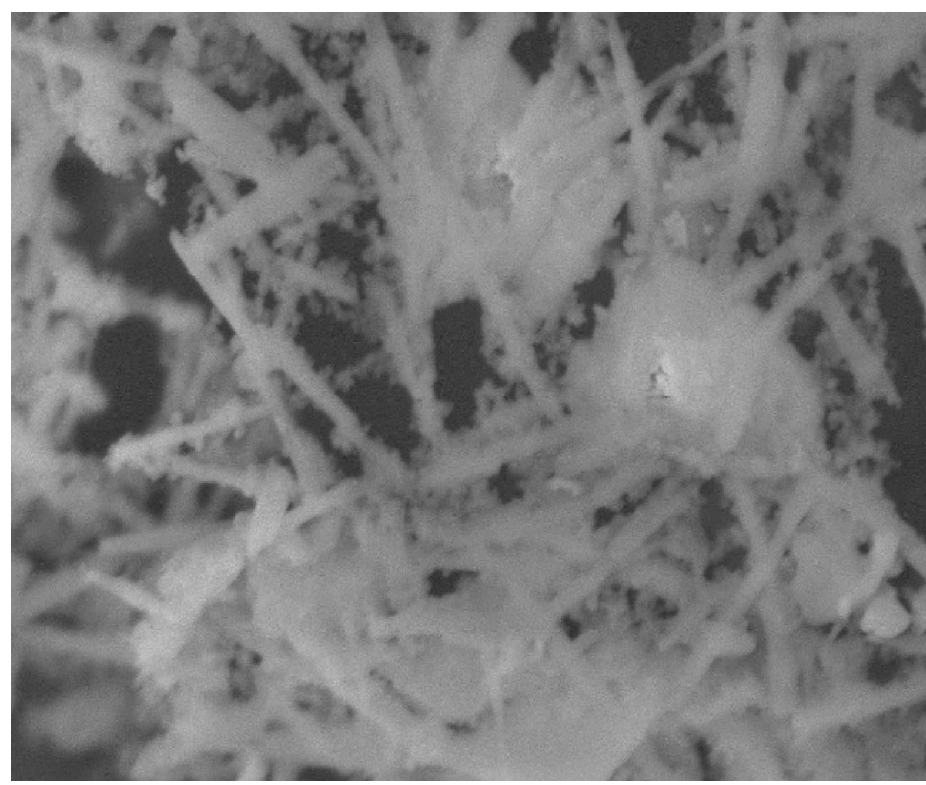

Figure 3: SEM micrographs of dried GONPs calcined at $400{ }^{\circ} \mathrm{C}(100 \mathrm{~nm})$

The high reaction temperature and sufficient time is favorable for the formation of large number of the cores in short time. The calcination of the production porous was useful to keep the porous shape [20]. The results are listed in Table 2 and it shows the variation of surface area, pore volume and pore size of GONPs with the calcination temperatures.

\section{DISCUSSION}

Ultraviolet (UV) spectroscopy confirmed the reduction of $\mathrm{AuCl}_{4}$ to porous GONPs. Metal nanoparticles such as silver and gold have free electrons, which gives rise to surface plasmon resonance (SPR) absorption band. The characteristic surface plasmon resonance band of GONPs was observed and exhibited an absorption band at $577.06 \mathrm{~nm}$ and the sharp peak indicates that the particles were monodispersed and were in nano size.

For more characterization of porous GONPs, Dynamic light scattering (DLS) zetasizer was employed for the measurement of the average particles size. The particles size of the green nanoparticles was variable and had an average value of $36 \mathrm{~nm}$. The polydispersity index (PDI) was dimensionless with values between 0 and 1 , the values with 0.10 or less are considered highly monodispersed [21]. DLS results of porous GONPs exhibited the PDI as 0.157 , which refers to mono-dispersity of nanoparticles which gives high stability to nanoparticles over a long time. This result was in agreement with the results of UV-vis absorption spectra.

Noble metals, such as gold and silver possess high conductivity and operational stability and require high temperature and vacuum deposition. Gold nanoparticles have been successfully explored as a low-temperature, high-conductivity alternative [22]. Zeta potential shows the electrical properties of nano-polymer porous gold oxide, which has electrical conductivity of 6 $\mathrm{mS} / \mathrm{cm}$. In the method used in the present study, the zeta potential was $26 \mathrm{mV}$ with positively charged nanoparticles. The higher zeta-potential value is a key parameter to maintain the stability of suspension because causes a repulsive force and keeps the gold nanoparticles away from each other.

Table 2: Relationship between the calcination temperatures and particle properties of porous GONPs

\begin{tabular}{lcccc}
\hline Parameter & \multicolumn{4}{c}{ Calcination temperature } \\
\cline { 2 - 5 } & $\mathbf{4 0 0}{ }^{\circ} \boldsymbol{C}$ & $\mathbf{5 0 0}{ }^{\circ} \boldsymbol{C}$ & $\mathbf{6 0 0}{ }^{\circ} \boldsymbol{C}$ & $\mathbf{7 0 0}^{\circ} \boldsymbol{C}$ \\
\hline Surface area $\left(\mathrm{m}^{2} / \mathrm{g}\right)$ & 0.0766 & 0.179 & 1.8708 & 146.706 \\
Pore volume $(\mathrm{cm} / \mathrm{g})$ & 0.000303 & 0.002181 & 0.007490 & 0.603574 \\
Pore size $(\mathrm{nm})$ & 22.3257 & 59.6814 & 21.4183 & 17.0949 \\
\hline
\end{tabular}


The result of TEM study gives a clear indication regarding the shape, size and distribution of GONPs. The surface morphology of the particles is porous, spherical, cylindrical, rod, cluster shapes and the display is highly dispersed. The particles were well-dispersed in solution at optimized gold salt concentration, which is due to efficient activity of both reducing and capping agents [23]. Variations in size can also be seen in UV-vis spectra (Fig. 1) as SPR bands shift towards longer wavelengths which indicate increased size of synthesized particles [24].

Among all the microscopic methods of analysis in literature, SEM and EDS are the most widely tools used to characterize the surface morphology of nano-polymer porous gold oxide. Several bright, cylindrical, rod-like, spherical, and cluster-shaped particles were observed in the SEM images.

The EDS spectra of GONPs showed peaks for gold and oxygen elements, which confirm the occupancy of GONPs, and a peak of zinc element which may have been produced from the biomolecules that skipped to the surface of the nanoparticles.

Results show that the calcined material at $700{ }^{\circ} \mathrm{C}$ results in a larger surface area and pore volume in comparison with the material calcined at 400 to $600{ }^{\circ} \mathrm{C}$. An increase in these parameters may be associated with a change in particle size as well as the removal of impurities and water molecules. GONPs exhibited smaller pore size at 600 and $700{ }^{\circ} \mathrm{C}$; which might be due to the blocking effects of the pore network.

\section{CONCLUSION}

A new environment-friendly approach to prepare nanopolymer porous gold oxide nanoparticles under simple conditions using sol-gel technology has been successfully developed in this study. The GONPs obtained are unique due to their high surface area $\left(146.706 \mathrm{~m}^{2} / \mathrm{g}\right)$ and high conductivity which makes them ideal for wide applications. This new approach may open a new window for the fabrication of various metal nano-structures with novel morphologies and varied applications.

\section{ACKNOWLEDGEMENT}

The authors extend their appreciation to the Deanship of Scientific Research at King Saud University for funding this work through Research Group no. RGP-VFF-278.

\section{REFERENCES}

1. Lu GQ, Zhao XS. Nanoporous Material - An overview. Nanoporous Materials: Science and Engineering. Imperial College Press 2004; Chapter 1.

2. Caixia $X$, Jixin $S$, Xiaohong $X$, Pengpeng T. Hongjuan $Z$, Fang T. Yi D. Low Temperature CO Oxidation over Unsupported Nanoporous Gold. J Am Chen: Soc 2007: 129:42-43.

3. Caixia X. Xiaohong $X$, Jixin S. Ding $Y$. Research on unsupported nanoporous gold catalyst for CO oxidation. J Catal 2007; 252: 243-248.

4. Chuanfang Y. Falong J, Zhihui A. Lizhi Z. Direct Oxidation of Methanol on Self-Supported Nanoporous Gold Film Electrodes with High Catalytic Activity and Stability. Chem Mater 2007: 19: 6065-6067.

5. Zielasek V, Jurgens B, Schulz C, Biener J. Biener Monika M. Hamza Alex V. Baumer M. Gold Catalysts: Nanoporous Gold Foams. Angew Chem Int Ed 2006: 45: 8241-8244.

6. Huang S, LiU X, Li Q, Meng M, Long T, Wang $H$, Jiang $Z$. The preparation of nanoporous sold electrodes by electrochemical alloying / dealloying process at room temperature and its properties. Materials Letters 2010: 64: 2296-2298.

7. Eitenberg RW, Andraka B, Takano Y. Prospects of porous gold as a low-temperature heat exchanger for liquid and solid helium. Phys B Condens Matter 2000: 284-288: 2022-2023.

8. Weissmuller J, Newman R, Jin H. Hodge A, Kysar J. Mater. Nanoporous Metals by Alloy Corrosion: Formation and Mechanical Propeities. Res. Soc. Bull 2009: 34: 577-586.

9. Ding Y, Chen M. Mater. Res. Soc. Bull 2009; 34 (8): 569576

10. Erkin S, Michael LR, Matthew RB. Nanoporous Gold: Fabrication, Characterization, and Applications. Materials 2009: 2: 2188-2215.

11. Yanping $D$, Wei $H$, Xin $C$, Zelin L. Facile fabrication of nanoporous gold film electrodes. Electrochem Commuu 2008: 10: 810-813.

12. Wei $Z$, Jmg-Juan $X$, Chuan-Guo $S$, Hong-Yuan $C$. Electrochem Commun 2006: 8: 773.

13. Alessia $\quad M$, Abbas $M$, Donald M, Michael B. Co. Sens Actuators B 2007: 12: 262-268.

14. Lang $X Y$, Guo $H$, Chen $L Y$, Kudo A, Yu JS, Zhang $W$. hioue A, Chen MW. Novel Nanoporous Au-Pd Alloy with High Catalytic Activity and Excellent Electrochemical Stability. J Phys Chem C 2010: 114:2600-3.

15. Huang Jing $F$, Sun W. Fabrication and surface Functionalization of Nanoporous Gold by Electrochemical Alloying/Dealloying of $\mathrm{Au}-\mathrm{Zn}$ in an ionic liquid, and the Self-Assembly of L- Cysteine Monolayers. Adv Fund Mater 2005: 15:989-994.

16. Fa long J, Chuan-fang Y, Zhihui A, Lizhi Z. Fabrication of Nanoporous Gold Films Electrodes with Ultrahigh Surface Area and Electrochemical Activity, Chem Mater 2007: 19:3648-3653.

Trop J Pharm Res, October 2015; 14(10): 1767 
17. Li YL, Song Yan-Y, Yang C, Xmg-Hua X. Hydrogen bubble dynamic template synthesis of porous gold for nonenzymatic electrochemical detection of glucose. Electrochem Cominim 2007: 9: 981 S.

18. Bartlett PN, Baumberg J J, Birkin Peter R, Ghanem MA, Netti MC. Highly Ordered Macroporous Gold and Platinum Films Formed by Electrochemical Desposition Through Templates Assembled From Submicron Diameter Monodisperse Polystyrene Spheres. Chem Mater 2002; 14:2199-208.

19. Huang S, Liu X, Li Q, Meng M, Long T, Wang H, Jiang Z. Mater Lett 2010: 64: 2296-2298.

20. Xue LZ, Ai Dong Z, Mi Ying J. Preparation and Characterization of Nano-polymer Porous $\mathrm{MgO}$. Chinese Chem Lett 2004: 11: 1342-1344.

21. Dynamic Light Scattering Common Terms Defined, Malvern Instruments Inform White Paper; 2011.
Available at: http://www.malvern.com/en/support/res ourcecenter/Whitepapers/WP111214DLSTermsDefined. aspx

22. Yuning L, Yiliang W, Beng SO. Facile Synthesis of Silver Nanopaiticles Useful for Fabrication of High-Conductivity Elements for Printed Electronics. J. Am. Chem. Soc 2005: 127: 3266-3267.

23. Malarkodi C, Rajeshkumar S, Vanaja M et al. Eco-friendly synthesis and characterization of gold nanoparticles using Klebsiella pneumoniae. J Nanostruct Chem(2013) : 3:30. doi:10.1186/2193-8865-3-30.

24. Huang J, Li Q, Sun $D$ et al. Biosynthesis of silver and gold nanoparticles by novel sundried Cinnamomum camphora leaf. Nanotechnology 2007: 18:104-105. doi:10.1088/0957-4484/18/10/105104. 